\title{
Seeded Multimode Quasi-Phase-Matching in All-Optically Poled Silicon Nitride Waveguides
}

\author{
Ozan Yakar ${ }^{1}$, Edgars Nitiss $^{1}$, Camille-Sophie Brès ${ }^{1, *}$ \\ ${ }^{1}$ Ecole Polytechnique Fédérale de Lausanne, Photonic Systems Laboratory (PHOSL), STI-IEL, Station 11, \\ CH-1015 Lausanne, Switzerland \\ *camille.bres@epfl.ch
}

\begin{abstract}
We demonstrate seeding-enabled all-optical poling of silicon nitride waveguides as short as $5 \mathrm{~mm}$, and account for quasi-phase-matching (QPM) inside the waveguide between the fundamental mode of pump and higher order second-harmonic modes. (1) 2021 The Author(s)
\end{abstract}

\section{Introduction}

Integrated silicon nitride $\left(\mathrm{Si}_{3} \mathrm{~N}_{4}\right)$ waveguides provide a flexible platform for scalable, low-cost $\chi^{(3)}$ nonlinear effects, and has been utilised for several applications such as four-wave mixing and supercontinuum generation. However, it lacks second-order nonlinearity due to its isotropic nature. Recently, it was shown that an effective $\chi^{(2)}$ nonlinearity can be inscribed through all-optical poling [1]. Here, only the optical fields are used to induce reconfigurable QPM in the waveguides. In earlier studies, bandwidth engineering, temperature tunability and stability of QPM were investigated. However in previous reports, the grating length was never longer than half of the total waveguide length . In this work, we show we can all-optically inscribe gratings in shorter waveguides and can increase the grating length using seeding - injection of external SH light with its pump. We also show the mixing of modes resulting in inscriptions of gratings with varying periods at the same position using two-photon (TP) imaging and spectral properties of the SH generation.

\section{Experiment and Results}

The waveguides are prepared with the photonic Damascene process [2] on a single chip. $\mathrm{The} \mathrm{Si}_{3} \mathrm{~N}_{4}$ waveguides are buried in $\mathrm{SiO}_{2}$ with input and output tapers, folded in meanders with bends having radius of $75 \mu \mathrm{m}$. We alloptically pole using a 1-ns, 5- $\mathrm{MHz}$ light at $1560 \mathrm{~nm}$ wavelength with a peak power up to $110 \mathrm{~W}$ described in [3]. Before the chip, we generate SH using an LBO crystal. We align polarizations of pump and SH and inject the two beams together to initiate the poling process as shown in Fig. 1a. In Fig 1b, the conversion efficiencies of waveguides of cross-section $1.9 \times 0.78 \mu \mathrm{m}^{2}$ with different lengths are shown. Seeding enabled poling of waveguides that are shorter than $22 \mathrm{~mm}$, which was not possible before relying only on the ns pump light source with powers less than $150 \mathrm{~W}$.

a)

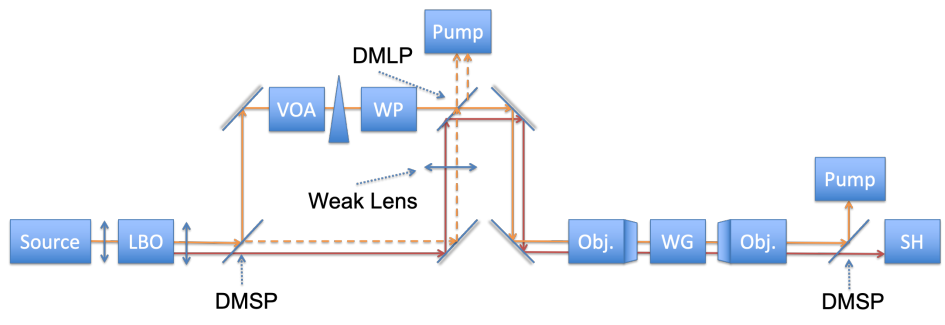

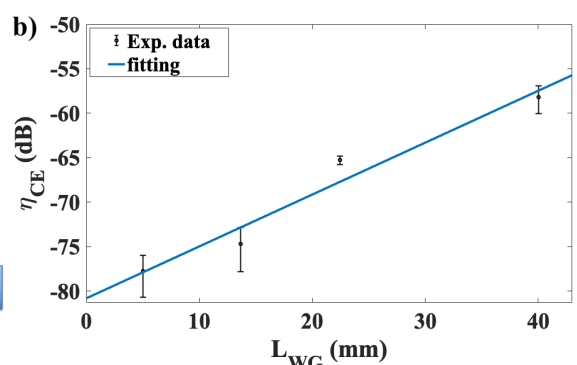

Fig. 1: a) Seeding setup. The TM pulsed light is sent to the LBO and TE SH is generated through 2 lenses then phases and polarizations are matched. To match the focal points of pump and SH a weak lens is used. The chip is all-optically poled using TE polarized light. We record the stability of the setup with the interferometer formed by the small transmission of pump from short-pass (DMSP) and reflection of pump from long-pass (DMLP) dichroic mirrors. b) Experimental data for CE and waveguide length $\left(L_{W G}\right)$ graph (black) and its fit (blue) for 1, 3, 5 and 9 meandered waveguides all-optically poled at $1560 \mathrm{~nm}$. The fit was $\eta_{C E}=0.5837 L_{W G}-80.82$.

The form of the $\chi^{(2)}$ grating in the waveguide due to the photogalvanic effect [4] is

$$
\chi^{(2)}=3 \chi^{(3)} E_{D C}(z) \sim \chi^{(3)}\left(E_{\omega}^{*}\right)^{2} E_{2 \omega} e^{-i \Delta \beta z}
$$


where $\mathrm{z}$ is the propagation direction, $\Delta \beta$ is the difference of the wavevectors of pump and SH, $E_{\omega}$ and $E_{2 \omega}$ are the amplitudes of the pump and SH fields, respectively. We observed the spatial distribution of charges (DC field) with TP imaging in Fig $2 \mathrm{c}$ and d. The grating image in the beginning of the waveguide in Fig. 2c shows that the grating
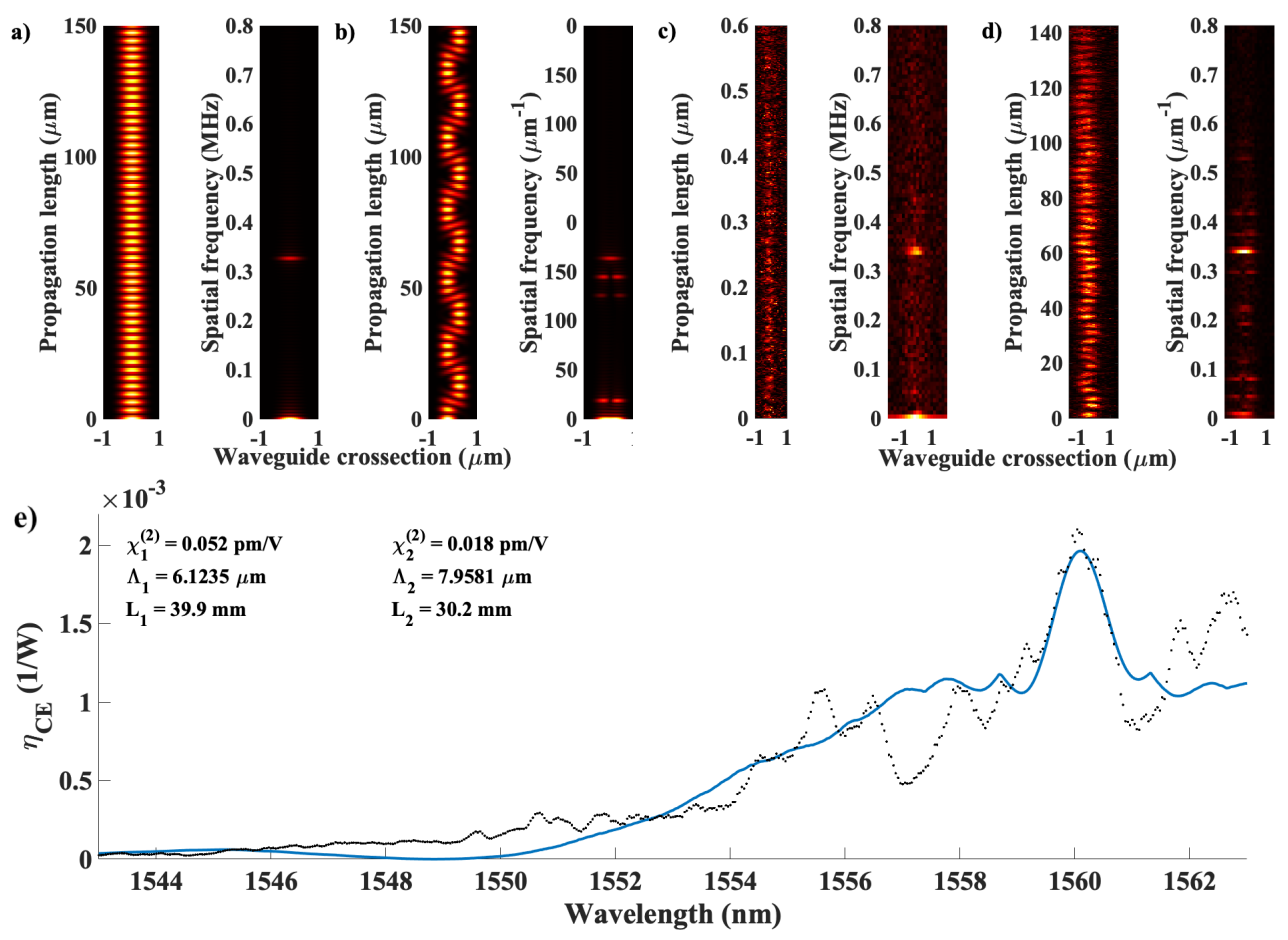

Fig. 2: Simulated magnitude of the DC field in the waveguide and its spectra for (a) fundamental mode of pump and fundamental mode of SH and (b) a mix of fundamental and second mode for the SH. TP images and spectral contents at (c) the beginning and (d) end of the waveguide. Contrasts of their spatial spectra were enhanced. e) Experimentally measured CE spectrum (black dots) with its fit (blue) of $40 \mathrm{~mm}$ waveguide of cross-section $1.9 \times 0.78 \mu \mathrm{m}^{2}$ folded in 9 meanders with $75 \mu \mathrm{m}$ bend radii. The effective nonlinearities $\left(\chi^{(2)}\right)$, grating periods $(\Lambda)$ and grating lengths $(L)$ for fundamental and second modes are obtained from the fits. The subscripts denote the mode number. The CE is measured to be $0.2 \% / W$.

is mostly due to the interaction of the fundamental modes of both pump and SH (Fig. 2a and c). Whereas, in the end of the waveguide we observe significant interaction of higher order SH modes. This serpentine like movement of the grating in Fig. 2d is due to the mixing of modes simulated in Fig. 2b. After $10 \mathrm{~mm}$, we observe the spectral contents of second mode of SH. This is in agreement with the double-sinc-squared fitted values for wavelength spectrum in Fig. 2e. Hence, bends with small curvatures allow for the energy transfer between the SH fundamental and higher order $\mathrm{SH}$ modes as predicted in ref. [3]. The remaining mismatch in the fit and experimental data in Fig. $2 \mathrm{e}$ is due to the involvement of other modes, which can be seen in Fig. 2 d.

We showed that waveguides as short as $5 \mathrm{~mm}$ can be poled with an externally seeded SH. In addition we can pole the entire waveguide length, which was not possible without seeding allowing for the enhanced interaction length. Also in large cross-section waveguides with small bend radii we gratings of higher order modes, accounting for the deviation of the CE spectrum shape from the expected sinc.

The funding was provided by ERC grant PISSARRO (ERC-2017-CoG 771647). The authors also thank Clemens Herkommer and Tobias J. Kippenberg from Laboratory of Photonics and Quantum Measurements at EPFL for the design and fabrication of the PIC used in this paper.

\section{References}

1. A. Billat, D. Grassani, M. H. Pfeiffer, S. Kharitonov, T. J. Kippenberg, and C.-S. Brès, "Large second harmonic generation enhancement in si 3 n 4 waveguides by all-optically induced quasi-phase-matching," Nat. communications 8, 1-7 (2017).

2. M. H. P. Pfeiffer, A. Kordts, V. Brasch, M. Zervas, M. Geiselmann, J. D. Jost, and T. J. Kippenberg, "Photonic damascene process for integrated high-q microresonator based nonlinear photonics," Optica 3, 20-25 (2016).

3. E. Nitiss, T. Liu, D. Grassani, M. Pfeiffer, T. J. Kippenberg, and C.-S. Bres, "Formation rules and dynamics of photoinduced $\chi$ (2) gratings in silicon nitride waveguides," ACS photonics 7, 147-153 (2019).

4. E. M. Dianov, P. G. Kazansky, and D. Y. Stepanov, "Photoinduced effects in optical waveguides," in International Workshop on Photoinduced Self-Organization Effects in Optical Fiber, , vol. 1516 (International Society for Optics and Photonics, 1991), pp. 81-98. 